\title{
An image-based children age range verification and classification based on facial features angle distribution and face shape elliptical ratio
}

\begin{abstract}
Verifying children are much easier than verifying adults, based on physical and body appearances. However it would be rather difficult to verify children's age referring only to their face properties. Therefore, this research presents an image-based method to classify children from adult and to verify children's age range. The method consists of two main stages; the process to distinguish children from adult based on input facial image and the process to verify children age range. The classification and verification algorithm was based on face shape elliptical ratio and facial features angle distribution. The angle that forms on human face images has been calculated based on selected facial features landmark points. The method was tested on FG-NET aging database. The classification of children from adults and the verification of children age range are implemented using SVM and Multi-SVM classification process. The results show an accuracy of classifying children from adults which are $92 \%$ more accurate than previous works.
\end{abstract}

Keyword: Age classification; Children age verification; Face shape elliptical ratio; Facial features angle distribution 